\title{
Existing clinical evidence on the use of cellular bone matrix grafts in spinal fusion: updated systematic review of the literature
}

\author{
Spencer C. Darveau, BA, Owen P. Leary, BS, Elijah M. Persad-Paisley, BA, \\ Elias A. Shaaya, MD, Adetokunbo A. Oyelese, MD, PhD, Jared S. Fridley, MD, \\ Prakash Sampath, MD, Joaquin Q. Camara-Quintana, MD, Ziya L. Gokaslan, MD, and \\ Tianyi Niu, MD \\ Department of Neurosurgery, The Warren Alpert School of Medicine, Brown University, Providence, Rhode Island
}

\begin{abstract}
OBJECTIVE Spinal fusion surgery is increasingly common; however, pseudarthrosis remains a common complication affecting as much as $15 \%$ of some patient populations. Currently, no clear consensus on the best bone graft materials to use exists. Recent advances have led to the development of cell-infused cellular bone matrices (CBMs), which contain living components such as mesenchymal stem cells (MSCs). Relatively few clinical outcome studies on the use of these grafts exist, although the number of such studies has increased in the last 5 years. In this study, the authors aimed to summarize and critically evaluate the existing clinical evidence on commercially available CBMs in spinal fusion and reported clinical outcomes.
\end{abstract}

METHODS The authors performed a systematic search of the MEDLINE and PubMed electronic databases for peerreviewed, English-language original articles (1970-2020) in which the articles' authors studied the clinical outcomes of CBMs in spinal fusion. The US National Library of Medicine electronic clinical trials database (www.ClinicalTrials.gov) was also searched for relevant ongoing clinical trials.

RESULTS Twelve published studies of 6 different CBM products met inclusion criteria: 5 studies of Osteocel Plus/Osteocel ( $n=354$ unique patients), 3 of Trinity Evolution $(n=114), 2$ of ViviGen $(n=171), 1$ of map3 $(n=41)$, and 1 of VIA Graft $(n=75)$. All studies reported high radiographic fusion success rates (range $87 \%-100 \%)$ using these CBMs. However, this literature was overwhelmingly limited to single-center, noncomparative studies. Seven studies disclosed industry funding or conflicts of interest (COls). There are 4 known trials of ViviGen ( 3 trials) and Bio4 (1 trial) that are ongoing. CONCLUSIONS CBMs are a promising technology with the potential of improving outcome after spinal fusion. However, while the number of studies conducted in humans has tripled since 2014, there is still insufficient evidence in the literature to recommend for or against CBMs relative to cheaper alternative materials. Comparative, multicenter trials and outcome registries free from industry COls are indicated.

https://thejns.org/doi/abs/10.3171/2021.3.FOCUS2173

KEYWORDS osteobiologics; cellular bone matrix; spinal fusion; mesenchymal stem cells; systematic literature review; conflicts of interest

$\mathrm{T}$ HE prevalence of spinal fusion in the United States has increased rapidly over the past several decades. ${ }^{1-3}$ Spinal fusions are employed to treat a range of spinal pathologies, including degenerative, infectious, neoplastic, and traumatic conditions. While imperfect, outcomes of spinal fusion procedures have improved alongside the de- velopment of new technologies to aid in spinal stabilization and fusion. Despite technological advances, pseudarthrosis occurs in up to $15 \%$ of patients, highlighting an opportunity for further improvement. ${ }^{4}$

Various allogenic and autogenic bone grafts have been used to augment spinal fusion. Iliac crest-derived auto-

ABBREVIATIONS ACDF = anterior cervical discectomy and fusion; ALIF = anterior lumbar interbody fusion; CBM = cellular bone matrix; COI = conflict of interest; ICBG = iliac crest-derived autograft; LLIF = lateral lumbar interbody fusion; MSC = mesenchymal stem cell; rhBMP = recombinant human bone morphogenetic protein; TLIF = transforaminal lumbar interbody fusion.

SUBMITTED January 31, 2021. ACCEPTED March 24, 2021.

INCLUDE WHEN CITING DOI: 10.3171/2021.3.FOCUS2173. 
grafts (ICBGs) became the gold standard due to their promotion of successful bone healing. However, the use of autografts requires separate surgical incisions, leading to increased operative time and donor site pain. ${ }^{5}$ In some fusion procedures, autogenic bone can be procured from structures within the same operative field. However, this is not always feasible or recommended and is occasionally contraindicated, for example, in spinal oncology cases in which tumor has invaded the bone. Relative to autograft, allograft substitutes such as demineralized bone matrix are associated with higher cost, rates of infection, and, in particular, poorer osteoinductivity. ${ }^{5,6}$ More recently, the use of allograft as a sole biological material in spinal fusion has declined in favor of other options such as recombinant human bone morphogenetic proteins (rhBMPs). While facilitating high fusion rates, concerns have been raised regarding the safety of rhBMP-2, with some studies finding an associated elevation in the risk of adverse events such as cage ejection and local edema. ${ }^{7.8}$ Reported perioperative adverse events associated with the use of rhBMP have also included heterotopic ossification, radiculitis, vertebral osteolysis, hematoma formation, interbody graft lucency, endplate decortication, and wound healing complications. ${ }^{9-13}$ Together, all of these disadvantages have motivated a renewed search for alternative bone substitutes. ${ }^{14}$

Over the past decade, investigators have increasingly explored ways of generating ideal bone grafts, which are defined as substances that promote three profusion cellular processes: osteoinduction, osteoconduction, and osteogenesis. ${ }^{15}$ Recent stem cell advances have led to a surge in the use of cellular bone matrices (CBMs), allogenic bone grafts containing live cellular components such as mesenchymal stem cells (MSCs) and osteoprogenitor cells. ${ }^{16}$ Bone matrices incorporating MSCs are potentially beneficial due to the ability of embedded MSCs to undergo postimplantation differentiation into native cellular components of bone. ${ }^{15}$ Several unique CBMs are now commercially available. At the time of the last comprehensive review of CBM use in spinal fusion, only 3 clinical trials had been published; that number has more than tripled in the last 6 years. ${ }^{16,17}$ This article summarizes and critically evaluates this rapidly expanding body of evidence and updates the spine surgery community on the available evidence surrounding commercially available CBM grafts in spinal fusions.

\section{Methods}

A systematic search of articles published in MEDLINE and PubMed databases (1970-2020) was performed. Specific PubMed search criteria included ((mesenchymal stem cells) OR (cellular bone matrix) OR (Osteocel) OR (ViviGen) OR (map3) OR (VIA Graft) OR (Trinity)) AND ((spinal fusion) OR ((spine) AND (fusion)) OR ((spine) AND (arthrodesis))). Identified articles were included if they were available in the English language and contributed new patients who had undergone spinal fusion using CBMs (Fig. 1). Level of evidence (I-V) was assessed for each article according to the GRADE (Grading of Recommendations Assessment, Development and Evaluation) working group and the AHRQ (Agency for Healthcare Research and Quality). ${ }^{18-20}$ The US National Library of Medicine electronic clinical trials database (www.ClinicalTrials. gov) was also searched for ongoing clinical trials of CBMs in spinal fusion. Studies conducted in preclinical models were omitted from this systematic review. ${ }^{16}$

\section{Results}

Twelve studies have evaluated CBMs in patients undergoing spinal fusion (Table 1). Published data on CBMs in spinal fusion have included studies of 5 commercially available products: Osteocel Plus/Osteocel (NuVasive), Trinity Evolution (Orthofix), ViviGen (LifeNet Health), map3 (RTI Surgical), and VIA Graft (Vivex Biomedical). Additionally, we identified 4 ongoing clinical trials evaluating 2 different CBMs in spinal fusion.

\section{Osteocel Plus}

Five studies reported fusion rates of the MSC-containing CBM Osteocel Plus (2011-2016). Kerr et al. retrospectively assessed the clinical effectiveness of Osteocel Plus for achieving radiological arthrodesis in patients undergoing lumbar interbody fusion at one $(69 \%)$ or two (31\%) levels. ${ }^{21}$ The authors studied 52 patients who underwent circumferential fusion (67\%), anterior lumbar interbody fusion (ALIF; 17\%), or lateral lumbar interbody fusion (LLIF; 16\%), and assessed radiological fusion based on plain radiographs and CT. Solid arthrodesis was achieved in $92.3 \%$ of patients at a median follow-up of 5 months. Age and smoking history increased time to arthrodesis. Kerr et al. concluded that Osteocel Plus is safe and effective for use in adults undergoing lumbar interbody fusion.

Ammerman et al. conducted another retrospective study of Osteocel Plus in 23 patients. ${ }^{22}$ The authors evaluated postoperative radiographs to define the rate of arthrodesis in patients who underwent minimally invasive transforaminal lumbar interbody fusion (TLIF) with Osteocel Plus at a total of 26 levels. Twenty-one patients (91.3\%) achieved radiographic evidence of solid bony arthrodesis at 24 levels $(92.3 \%)$ by the 12-month follow-up, with $6(26 \%)$ of the patients exhibiting clear interbody bone growth within 6 months postoperatively. The authors concluded that Osteocel Plus facilitates robust and reproducible lumbar interbody fusion.

Tohmeh et al. evaluated rates of arthrodesis in patients who underwent LLIF with Osteocel Plus at 12 months postoperatively. ${ }^{23}$ They included 40 patients who underwent interbody fusion at a total of 61 levels. Completeness of fusion was clinically assessed using a combination of $\mathrm{CT}$ scans and plain radiographs, with successful arthrodesis demonstrated at 55 levels (90.2\%), and the remaining 6 (9.8\%) demonstrating partial pseudarthrosis at 12 months postoperation. Tohmeh et al. concluded that Osteocel Plus facilitates successful lumbar interbody fusion.

Eastlack et al. prospectively evaluated 2-year outcomes of treatment with Osteocel Plus during one- or two-level anterior cervical discectomy and fusion (ACDF), including 249 levels across 182 patients. ${ }^{24}$ In this multicenter, nonrandomized study, fusion was evaluated using both CT and plain radiographs. The authors found that, at the 2-year follow-up, $92 \%$ of single-level procedures and $87 \%$ of to- 


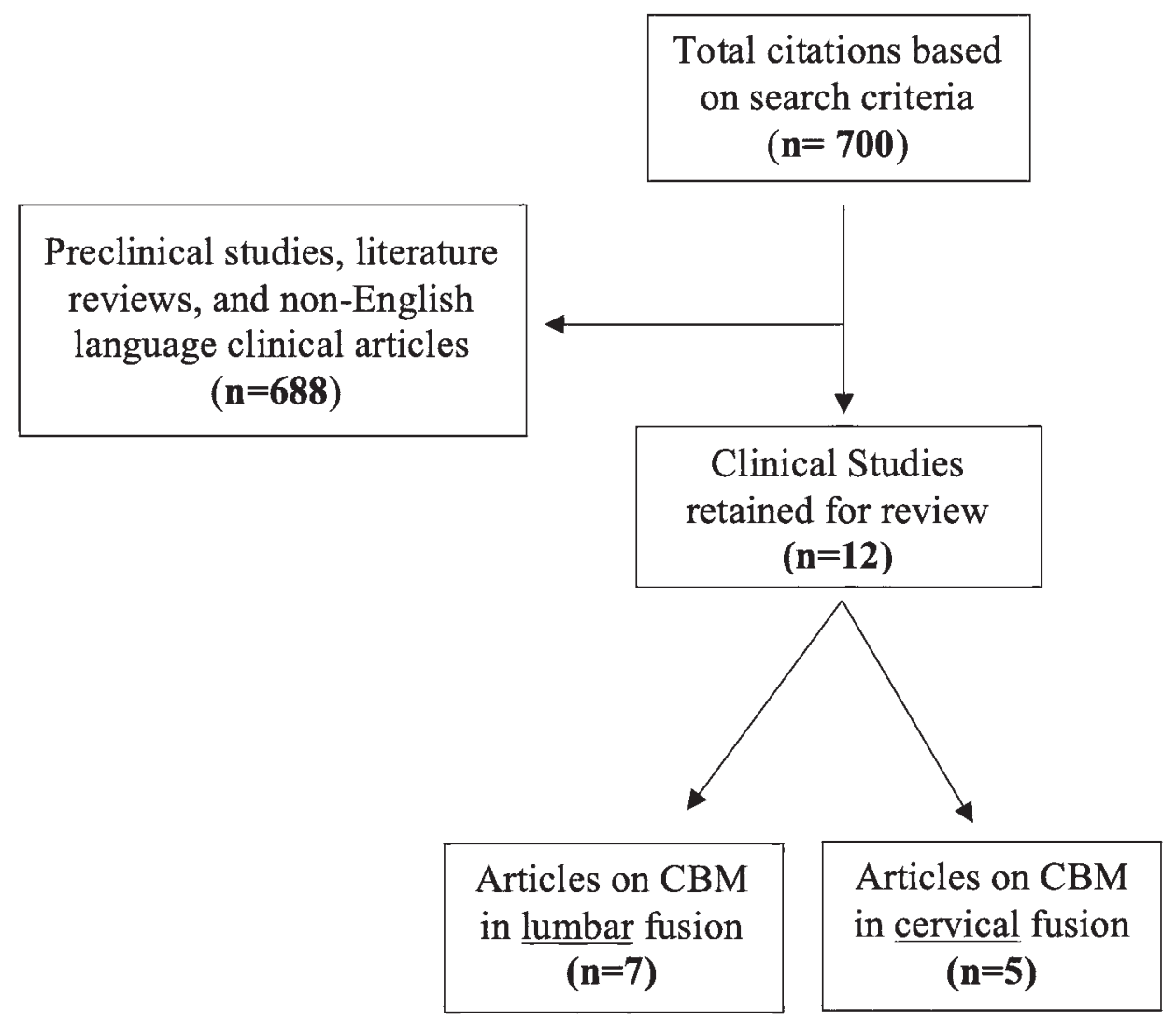

FIG. 1. Flowchart detailing results of comprehensive literature review using PubMed search criteria.

tal fused levels resulted in solid fusion, with no patients requiring revision for pseudarthrosis. For comparison, the single-level fusion rate has previously been reported to be 92.1\% for ACDF, and $97.1 \%$ for ACDF with anterior plating, based on a meta-analysis of the literature as of 2007.

Most recently, McAnany et al. studied a cohort of $57 \mathrm{pa}-$ tients who received allograft and Osteocel during ACDF with anterior plating and a matched control group of 57 patients who underwent the same procedure with standard allograft alone. ${ }^{26}$ There was no difference in age, body mass index, or comorbidity burden between groups. Of the 57 patients who underwent ACDF with Osteocel, 50 (87.7\%) demonstrated solid fusion on CT compared with $54(94.7 \%)$ in the control group. The authors concluded that Osteocel was associated with lower fusion rates compared with allograft alone in ACDF.

\section{Trinity Evolution}

Three studies (2016-2017) investigated fusion rates of patients who received Trinity Evolution, another MSCcontaining CBM. Musante et al. retrospectively evaluated 12-month radiographic and clinical outcomes of one- and two-level posterolateral lumbar fusion using Trinity Evolution in 43 patients. ${ }^{27}$ Complete arthrodesis was observed in $90.7 \%$ of participants and $89.4 \%$ of levels on 12-month postoperative radiographs. Patients deemed to be at higher risk of pseudarthrosis did not experience markedly lower fusion rates. The authors concluded that Trinity Evolution comprises a safe and efficacious replacement for autologous ICBG even among patients at risk for nonunion.

Vanichkachorn et al. studied the safety and efficacy of Trinity Evolution in a retrospective cohort of patients undergoing ACDF. ${ }^{28}$ They evaluated 31 patients who underwent single-level ACDF at 6 and 12 months postoperatively using $\mathrm{CT}$ and flexion-extension radiography. The overall reported fusion rate was $78.6 \%$ at 6 months and $93.5 \%$ at 12 months postoperatively. Vanichkachorn et al. concluded that patients undergoing single-level ACDF with Trinity Evolution had a high fusion rate without serious complications despite risk factors.

In a prospective, multicenter study, Peppers et al. assessed the radiographic and clinical outcomes of $40 \mathrm{pa}-$ tients who underwent two-level ACDF with Trinity Evolution. ${ }^{29}$ Twelve-month postoperative outcome was evaluated by flexion-extension radiography and CT to evaluate fusion success. Subject-wise and level-wise rates of successful arthrodesis were $89.4 \%$ and $93.4 \%$, respectively. Patients with more risk factors for pseudarthrosis demonstrated no significant decrease in spinal fusion rates. The authors concluded that Trinity Evolution presents a viable clinical alternative to non-CBM products.

\section{ViviGen}

ViviGen is a CBM graft that incorporates osteoblasts and osteocytes, rather than MSCs, within its osteoconductive matrix. Preclinical data have suggested that CBMs 


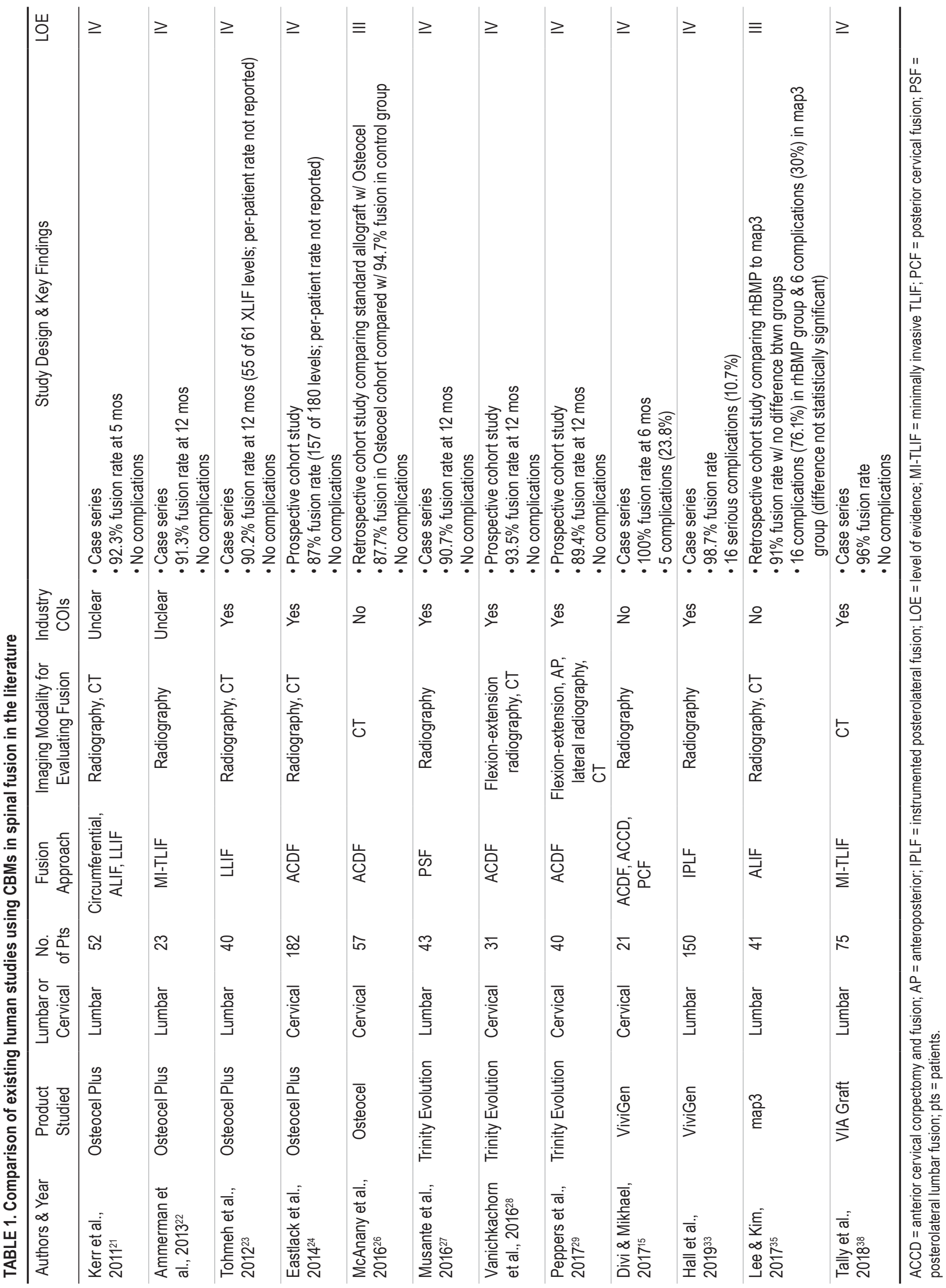


containing osteoprogenitor cells or other lineage-committed bone cells might promote better osteogenesis and increased calcium deposition compared with MSC-containing CBMs. ${ }^{30-32}$ Since the last comprehensive review of human CBM trials, the first two studies in humans of ViviGen in spine fusion have been published (2017 and 2019). ${ }^{16}$ Divi and Mikhael reported a series of 21 patients undergoing multilevel ACDF, anterior cervical corpectomy and fusion, or posterior cervical fusion, in which ViviGen was applied by a single surgeon. ${ }^{15}$ Patients underwent routine postoperative radiography, and the authors reported a $100 \%$ 12-month fusion rate with no reoperations. Sixteen patients had risk factors for poor fusion. Delayed wound healing was reported in 3 patients.

Hall et al. reported another series of 150 consecutive patients who underwent ViviGen-augmented instrumented posterolateral lumbar fusion performed by a single surgeon at 613 spinal levels. ${ }^{33}$ Patients were monitored postoperatively with at least radiography. The overall fusion rate was $98.7 \%$, with pseudarthrosis occurring in 2 patients $(1.3 \%)$. The authors noted the relative complexity of their population, citing an average of 4.1 levels treated and many patients having multiple comorbid risk factors for pseudarthrosis.

The NIH currently lists three registered clinical trials on the use of ViviGen in cervical or lumbar spinal fusion (identifiers NCT02814825, NCT04007094, and NCT03733626). The first, which is not yet recruiting, will be a multicenter study of 100 participants undergoing ACDF. The second is recruiting 50 patients undergoing posterolateral lumbar fusion. The third will be a randomized controlled trial of 40 participants undergoing posterolateral lumbar fusion receiving either ViviGen or autologous bone graft.

\section{Map3}

Another relatively new CBM alternative is map3, which contains multipotent adult progenitor cells intended to potentiate angiogenesis and osteogenesis at the fusion site. ${ }^{34}$ The first human study of map3 was published by Lee and Kim in 2017, in which 41 patients treated with ALIF received either map3 $(n=20)$ or rhBMP $(n=21)$, and the two groups were compared in terms of fusion and complication rates. ${ }^{35}$ The authors reported fusion rates of $92 \%$ in the rhBMP group and $90 \%$ in the map3 group, which was not a statistically significant difference. Significant improvement in Oswestry Disability Index scores was seen across both groups, and comorbidity profiles were similar. The rhBMP group experienced 16 complications $(8$ of which were due to postoperative radiculitis), and the map3 group experienced only 6 complications; however, the difference was not statistically significant.

\section{VIA Graft}

VIA Graft is composed of allogeneic cortical bone particles, MSCs, osteoprogenitor cells, and a polyampholytebased cryopreservative agent, which has been shown to be safer and more protective of the cell components than the dimethyl sulfoxide in other CBMs. ${ }^{36,37}$ The first study of VIA Graft use in humans was a retrospective case series by Tally et al. in 2018, in which 12-month fusion rates were evaluated for 75 patients who underwent minimally invasive TLIF. ${ }^{38}$ Fusion was assessed using CT. ${ }^{38}$ Ninetysix percent of patients and $96.5 \%$ of levels had achieved solid fusion at the 12-month follow-up. There were no perioperative complications. Tally et al. concluded that this high rate of fusion without complications supports the safety and efficacy of VIA Graft in TLIF.

\section{Other CBM Products}

Several other CBM grafts are also commercially available. AlloStem (AlloSource), Cellentra Viable Bone Cell Matrix (Zimmer Biomet), and Ovation (Osiris Therapeutics) utilize matrices infused with MSCs harvested from human donors and have been described in detail in a prior review. ${ }^{16}$ To our knowledge, there are no peer-reviewed clinical studies comparing or documenting outcomes using these specific products in spinal fusion. A fourth product, Bio4 (Stryker), has been promoted by the manufacturer as angiogenic (similar to map3) and incorporates MSCs, osteoprogenitor cells, and angiogenic growth factors. An open-label study of Bio4 in 20 patients undergoing ACDF is underway (identifier NCT03077204).

\section{Discussion}

The increasing prevalence of spinal fusion coupled with the desire to further reduce pseudarthrosis rates has prompted the investigation and use of alternative bone grafts. CBMs containing MSCs or osteoprogenitor cells have been recently explored as alternatives to ICBGs, but questions remain related to whether MSCs and other cells continue to be viable once CBMs are implanted in humans. While it is challenging to study these questions in humans, the efficacy of CBMs, relative to other existing biologic products, would best be demonstrated through comparative clinical study in well-defined spinal fusion cohorts.

Unfortunately, few such studies exist, and those that do harbor significant limitations. The goal of this literature review was to provide an updated critical evaluation of the existing evidence on the use of commercially available CBM grafts in spinal fusion. Notably, none of these products have been formally approved by the US FDA. Most products we discuss are considered by the manufacturers to meet criteria for HCT/P (human cells, tissues, and cellular and tissue-based products) exemption from federal $510(\mathrm{k})$ submission requirements. The applicability of this exemption has been disputed on a case-by-case basis in recent years; nevertheless, CBM grafts are still widely available commercially. The approval status of this market further underscores the need for frequent re-review of existing clinical evidence to help surgeons decide whether use of these products is appropriate on a case-by-case basis. This is already standard practice for the off-label use of other biological products; only a few of the rhBMP products, notably, osteogenic protein-1 (Stryker Biotech) and Infuse (Medtronic), are approved by the FDA, with $85 \%$ of primary spine procedures utilizing BMP products off label. ${ }^{39}$ Despite the high radiographic fusion rates associated with BMP, there have been concerns about the rare but serious neurological or structural complications 
that have been reported. ${ }^{40,41}$ The controversy that ensued extended to a third-party review of data that had been previously used to bring these products to market, which called into question the superiority of rhBMP compared with ICBG in standard fusion cases. ${ }^{42}$ The search for a viable alternative with more modest adverse effects led to the development of MSC-containing allografts and CBMs, but clinical data remain limited.

The two oldest CBMs included in this review are Osteocel Plus and Trinity Evolution. Of the 5 studies evaluating Osteocel Plus, 3 involved lumbar fusions and 2 involved cervical fusions. ${ }^{21-24,26}$ Fusion rates ranged from $87.0 \%$ at 2 years to $92.3 \%$ at 5 months, and no clinical complications were observed. ${ }^{21-24,26}$ The studies by Kerr et al. and Ammerman et al. did not disclose whether the authors had any conflicts of interest (COIs). ${ }^{21,22}$ Tohmeh et al. and Eastlack et al. reported COIs. ${ }^{23,24}$ The McAnany et al. study was not industry sponsored and is the first of its kind to compare a matched cohort assessing 12-month fusion rates in ACDF with and without CBM. ${ }^{26}$ Trinity Evolution was evaluated across 3 studies with similarly sized cohorts. All 3 disclosed COIs, including Orthofix's involvement in the design and interpretation of data in the study by Peppers et al. ${ }^{29}$ Fusion rates were similar to those for Osteocel Plus at 12 months after lumbar or cervical fusion. ${ }^{27-29}$ Collectively, these studies present only low-level evidence for the safety and efficacy of these products without direct comparison with alternatives. Results are further weakened by the fact that studies of fusion in the cervical and lumbar spine are not truly comparable; while overall cervical fusion rates range from $80 \%$ to $97 \%$, lumbar fusion rates range from $83 \%$ to $98 \%$, both dependent on approach, number of levels fused, and graft materials used. ${ }^{25,43}$ Further variation exists between fusion procedures within the cervical and lumbar groups; for example, Kerr et al. combined LLIF and ALIF in the same cohort. ${ }^{21}$

Compared with Osteocel Plus and Trinity Evolution, ViviGen, map3, and VIA Graft are relative newcomers. As such, very little safety and efficacy data exist for patients undergoing spinal fusion with these products. One of two studies of ViviGen reported COIs. Both studies' reported fusion rates were among the highest documented in this review ( $>98 \%$ ), but postoperative complication rates (up to $23.8 \%$ ) were also higher than those reported in the literature for both cervical $(13 \%-19 \%)$ and lumbar $(9 \%-17 \%)$ fusion using non-CBM materials. ${ }^{15,33,44-46}$ For map3, only a single retrospective cohort study was identified. Lee and Kim found no difference in lumbar fusion rate (91\%) between map3 and rhBMP. ${ }^{35}$ Both products were associated with symptomatic improvement, and the study was conducted free from COIs. The complication rate was higher in the rhBMP group (76.1\%) than in the map3 group $(30 \%)$. Findings were consistent with prior published fusion rates for both rhBMP and non-CBM fusion materials, and complication rates of map3 were comparable to those reported for ViviGen. This study is a commendable example of a head-to-head comparative study between CBM and nonCBM fusion alternatives. Finally, only one case series used VIA Graft in minimally invasive TLIF. Tally et al. found a very high fusion rate (96\%) and no perioperative or longterm complications. ${ }^{38}$ Two of the authors are consultants for Vivex but received no compensation for this project. For these newer CBM grafts, clinical evidence is lacking, and comparative studies are needed.

\section{Current Limitations to Implementation of CBMs}

While CBMs remain a promising alternative to autograft in spinal fusion, there is a marked dearth of clinical studies comparing the various commercially available CBMs in terms of fusion rates, costs, and complication profiles when applied to augmentation of cervical or lumbar spinal fusion. In this review, we found that while the volume of literature has more than doubled since the last comprehensive review on this topic, ${ }^{16}$ most of the existing studies of the use of these products in humans, including ongoing clinical trials of which we are presently aware, are still limited to relatively small single-center studies examining outcomes without comparator groups and often with industry support from CBM manufacturers. As such, the level of evidence across all existing clinical literature on the use of CBMs in spinal fusion remains quite low (Table 1). ${ }^{47}$ There also appear to be no studies comparing costs, either between available CBM products or between CBMs and other allograft alternatives.

Some inferences regarding interproduct comparison can be drawn from well-conducted animal studies. One recent study compared 6-week fusion outcomes of 6 commercially available human CBMs (Cellentra, Trinity, ViviGen, Osteocel, map3, and Bio4) after use for augmenting spinal fusion in athymic rats. ${ }^{48}$ Analysis of microCT performed in 15 euthanized animals revealed stable fusion in $11,8,2,0,0$, and 0 for the $6 \mathrm{CBM}$ products, respectively. ${ }^{48}$ The authors concluded that the Trinity and Cellentra products achieved significantly better fusion rates in this particular animal model. A second rodent study performed by Hayashi et al. compared Trinity Evolution with nonMSC-containing demineralized bone matrix alternatives and found that, while Trinity Evolution provided a significantly higher rate of fusion as evaluated with radiography at 8 weeks, the fusion rate was not significantly different between products when evaluated by microCT. ${ }^{49}$ Results have implications for human trials, suggesting that fusion rates should optimally be evaluated using CT. Our review found that, while many studies indeed used CT to evaluate fusion, others relied on radiography, suggesting a possible tendency to overestimate fusion rates in the existing CBM literature (Table 1).

The main barriers to conducting prospective randomized studies on CBMs in spinal fusion appear to be related to 1) the lack of FDA approval for these products and 2) the reliance of existing literature on industry sponsorship for completing clinical outcome studies. Furthermore, the high cost of these products relative to more widely used and FDA-approved alternatives, such as demineralized bone matrix or rhBMP, may be compounded by a lack of transparency regarding cost data. The majority of both published studies and ongoing trials are industry sponsored, introducing COIs and further potential for bias. ${ }^{50,51}$ As spine surgeons remain in pursuit of higher fusion rates and lower complication rates across a broadening and increasingly complex spinal fusion population, future studies providing high-quality safety and efficacy data on the 
use of CBMs would be welcomed and potentially very impactful.

\section{Conclusions}

Our review of existing literature documents a growing body of evidence that CBMs are a promising technology in spinal fusion. The number of published clinical studies has tripled in the last 6 years, and the number of different CBMs evaluated has increased from 1 to 5, with 4 additional clinical trials currently underway. Results generally suggest high fusion rates and low complication rates, but efficacy data remain based on a relatively small number of single-institution series with a high prevalence of industry COIs. Therefore, current evidence remains insufficient to make a definitive statement on the safety, efficacy, or costeffectiveness of any of the products, and the decision to use them should be made on an individual basis by the surgeon after considering these limitations.

\section{References}

1. Tarpada SP, Morris MT, Burton DA. Spinal fusion surgery: a historical perspective. J Orthop. 2016;14(1):134-136.

2. Rajaee SS, Bae HW, Kanim LEA, Delamarter RB. Spinal fusion in the United States: analysis of trends from 1998 to 2008. Spine (Phila Pa 1976). 2012;37(1):67-76.

3. Martin BI, Mirza SK, Spina N, et al. Trends in lumbar fusion procedure rates and associated hospital costs for degenerative spinal diseases in the United States, 2004 to 2015. Spine (Phila Pa 1976). 2019;44(5):369-376.

4. Hsu WK, Wang JC. The use of bone morphogenetic protein in spine fusion. Spine J. 2008;8(3):419-425.

5. Kerezoudis P, Alvi MA, Freedman BA, et al. Utilization trends of recombinant human bone morphogenetic protein in the United States. Spine (Phila Pa 1976). Published online December 31, 2020. doi:10.1097/BRS.0000000000003919

6. Roberts TT, Rosenbaum AJ. Bone grafts, bone substitutes and orthobiologics: the bridge between basic science and clinical advancements in fracture healing. Organogenesis. 2012;8(4):114-124.

7. Singh K, Ahmadinia K, Park DK, et al. Complications of spinal fusion with utilization of bone morphogenetic protein: a systematic review of the literature. Spine (Phila Pa 1976). 2014;39(1):91-101.

8. Adams CL, Ogden K, Robertson IK, et al. Effectiveness and safety of recombinant human bone morphogenetic protein-2 versus local bone graft in primary lumbar interbody fusions. Spine (Phila Pa 1976). 2014;39(2):164-171.

9. Arnold PM, Anderson KK, Selim A, et al. Heterotopic ossification following single-level anterior cervical discectomy and fusion: results from the prospective, multicenter, historically controlled trial comparing allograft to an optimized dose of rhBMP-2. J Neurosurg Spine. 2016;25(3):292-302.

10. Villavicencio AT, Burneikiene S. RhBMP-2-induced radiculitis in patients undergoing transforaminal lumbar interbody fusion: relationship to dose. Spine J. 2016;16(10):1208-1213.

11. Balseiro S, Nottmeier EW. Vertebral osteolysis originating from subchondral cyst end plate defects in transforaminal lumbar interbody fusion using rhBMP-2. Report of two cases. Spine J. 2010;10(7):e6-e10.

12. Tannoury CA, An HS. Complications with the use of bone morphogenetic protein 2 (BMP-2) in spine surgery. Spine $J$. 2014;14(3):552-559.

13. Smoljanovic T, Cimic M, Bojanic I. Aggressive end plate decortication as a cause of osteolysis after rhBMP-2 use in cervical spine interbody fusion. Spine J. 2010;10(2):187-188.
14. Sohn HS, Oh JK. Review of bone graft and bone substitutes with an emphasis on fracture surgeries. Biomater Res. 2019;23(1):9.

15. Divi SN, Mikhael MM. Use of allogenic mesenchymal cellular bone matrix in anterior and posterior cervical spinal fusion: a case series of 21 patients. Asian Spine $J$. 2017;11(3):454-462.

16. Skovrlj B, Guzman JZ, Al Maaieh M, et al. Cellular bone matrices: viable stem cell-containing bone graft substitutes. Spine J. 2014;14(11):2763-2772.

17. Hsieh PC, Buser Z, Skelly AC, et al. Allogenic stem cells in spinal fusion: a systematic review. Global Spine J. 2019;9(1) (suppl):22S-38S.

18. Wright JG, Swiontkowski MF, Heckman JD. Introducing levels of evidence to the journal. J Bone Joint Surg Am. 2003;85(1):1-3.

19. West S, King V, Carey TS, et al. Systems to rate the strength of scientific evidence. Evid Rep Technol Assess (Summ). 2002;47:1-11.

20. Atkins D, Best D, Briss PA, et al. Grading quality of evidence and strength of recommendations. $B M J$. 2004;328(7454):1490.

21. Kerr EJ III, Jawahar A, Wooten T, et al. The use of osteoconductive stem-cells allograft in lumbar interbody fusion procedures: an alternative to recombinant human bone morphogenetic protein. J Surg Orthop Adv. 2011;20(3):193-197.

22. Ammerman JM, Libricz J, Ammerman MD. The role of Osteocel Plus as a fusion substrate in minimally invasive instrumented transforaminal lumbar interbody fusion. Clin Neurol Neurosurg. 2013;115(7):991-994.

23. Tohmeh AG, Watson B, Tohmeh M, Zielinski XJ. Allograft cellular bone matrix in extreme lateral interbody fusion: preliminary radiographic and clinical outcomes. ScientificWorldJournal. 2012;2012:263637.

24. Eastlack RK, Garfin SR, Brown CR, Meyer SC. Osteocel Plus cellular allograft in anterior cervical discectomy and fusion: evaluation of clinical and radiographic outcomes from a prospective multicenter study. Spine (Phila $\mathrm{Pa}$ 1976). 2014;39(22):E1331-E1337.

25. Fraser JF, Härtl R. Anterior approaches to fusion of the cervical spine: a metaanalysis of fusion rates. J Neurosurg Spine. 2007;6(4):298-303.

26. McAnany SJ, Ahn J, Elboghdady IM, et al. Mesenchymal stem cell allograft as a fusion adjunct in one- and two-level anterior cervical discectomy and fusion: a matched cohort analysis. Spine J. 2016;16(2):163-167.

27. Musante DB, Firtha ME, Atkinson BL, et al. Clinical evaluation of an allogeneic bone matrix containing viable osteogenic cells in patients undergoing one- and two-level posterolateral lumbar arthrodesis with decompressive laminectomy. $J$ Orthop Surg Res. 2016;11(1):63.

28. Vanichkachorn J, Peppers T, Bullard D, et al. A prospective clinical and radiographic 12-month outcome study of patients undergoing single-level anterior cervical discectomy and fusion for symptomatic cervical degenerative disc disease utilizing a novel viable allogeneic, cancellous, bone matrix (Trinity Evolution ${ }^{\mathrm{TM}}$ ) with a comparison to historical controls. Eur Spine J. 2016;25(7):2233-2238.

29. Peppers TA, Bullard DE, Vanichkachorn JS, et al. Prospective clinical and radiographic evaluation of an allogeneic bone matrix containing stem cells (Trinity Evolution ${ }^{\circledR}$ Viable Cellular Bone Matrix) in patients undergoing two-level anterior cervical discectomy and fusion. J Orthop Surg Res. 2017;12(1):67.

30. Birmingham E, Niebur GL, McHugh PE, et al. Osteogenic differentiation of mesenchymal stem cells is regulated by osteocyte and osteoblast cells in a simplified bone niche. Eur Cell Mater. 2012;23:13-27.

31. Ghanaati S, Barbeck M, Hilbig U, et al. An injectable bone 
substitute composed of beta-tricalcium phosphate granules, methylcellulose and hyaluronic acid inhibits connective tissue influx into its implantation bed in vivo. Acta Biomater. 2011;7(11):4018-4028.

32. Tortelli F, Tasso R, Loiacono F, Cancedda R. The development of tissue-engineered bone of different origin through endochondral and intramembranous ossification following the implantation of mesenchymal stem cells and osteoblasts in a murine model. Biomaterials. 2010;31(2):242-249.

33. Hall JF, McLean JB, Jones SM, et al. Multilevel instrumented posterolateral lumbar spine fusion with an allogeneic cellular bone graft. J Orthop Surg Res. 2019;14(1):372.

34. LoGuidice A, Houlihan A, Deans R. Multipotent adult progenitor cells on an allograft scaffold facilitate the bone repair process. J Tissue Eng. 2016;7:2041731416656148.

35. Lee DD, Kim JY. A comparison of radiographic and clinical outcomes of anterior lumbar interbody fusion performed with either a cellular bone allograft containing multipotent adult progenitor cells or recombinant human bone morphogenetic protein-2. J Orthop Surg Res. 2017;12(1):126.

36. Son JH, Kim KH, Nam YK, et al. Optimization of cryoprotectants for cryopreservation of rat hepatocyte. Biotechnol Lett. 2004;26(10):829-833.

37. Men H, Agca Y, Critser ES, Critser JK. Beneficial effects of serum supplementation during in vitro production of porcine embryos on their ability to survive cryopreservation by open pulled straw vitrification. Theriogenology. 2005;64(6):1340 1349.

38. Tally WC, Temple HT, Subhawong TY, Ganey T. Transforaminal lumbar interbody fusion with viable allograft: 75 consecutive cases at 12-month follow-up. Int J Spine Surg. 2018;12(1):76-84.

39. Ong KL, Villarraga ML, Lau E, et al. Off-label use of bone morphogenetic proteins in the United States using administrative data. Spine (Phila Pa 1976). 2010;35(19):1794-1800.

40. Rihn JA, Patel R, Makda J, et al. Complications associated with single-level transforaminal lumbar interbody fusion. Spine J. 2009;9(8):623-629.

41. Meisel HJ, Schnöring M, Hohaus C, et al. Posterior lumbar interbody fusion using rhBMP-2. Eur Spine J. 2008;17(12):1735-1744.

42. Hustedt JW, Blizzard DJ. The controversy surrounding bone morphogenetic proteins in the spine: a review of current research. Yale J Biol Med. 2014;87(4):549-561.

43. Galimberti F, Lubelski D, Healy AT, et al. A systematic review of lumbar fusion rates with and without the use of rhBMP-2. Spine (Phila Pa 1976). 2015;40(14):1132-1139.

44. Epstein NE. Complications due to the use of BMP/INFUSE in spine surgery: the evidence continues to mount. Surg Neurol Int. 2013;4(5)(suppl 5):S343-S352.
45. Kalanithi PS, Patil CG, Boakye M. National complication rates and disposition after posterior lumbar fusion for acquired spondylolisthesis. Spine (Phila Pa 1976). 2009;34(18):1963-1969.

46. de Kunder SL, van Kuijk SMJ, Rijkers K, et al. Transforaminal lumbar interbody fusion (TLIF) versus posterior lumbar interbody fusion (PLIF) in lumbar spondylolisthesis: a systematic review and meta-analysis. Spine J. 2017;17(11):17121721.

47. Wang JC, Yoon ST, Brodke DS, et al. Development of AOSpine BOnE (Bone Osteobiologics and Evidence) Classification. Global Spine J. 2020;10(7):871-874.

48. Lin C, Zhang N, Waldorff EI, et al. Comparing cellular bone matrices for posterolateral spinal fusion in a rat model. JOR Spine. 2020;3(2):e1084.

49. Hayashi T, Lord EL, Suzuki A, et al. A comparison of commercially available demineralized bone matrices with and without human mesenchymal stem cells in a rodent spinal fusion model. J Neurosurg Spine. 2016;25(1):133-137.

50. Amiri AR, Kanesalingam K, Cro S, Casey ATH. Does source of funding and conflict of interest influence the outcome and quality of spinal research? Spine J. 2014;14(2):308-314.

51. Fenton JJ, Mirza SK, Lahad A, et al. Variation in reported safety of lumbar interbody fusion: influence of industrial sponsorship and other study characteristics. Spine (Phila Pa 1976). 2007;32(4):471-480.

\section{Disclosures}

The authors report no conflict of interest concerning the materials or methods used in this study or the findings specified in this paper.

\section{Author Contributions}

Conception and design: Darveau, Leary, Niu. Acquisition of data: Darveau. Analysis and interpretation of data: Darveau, Niu. Drafting the article: Darveau, Leary, Persad-Paisley, Shaaya, Niu. Critically revising the article: all authors. Reviewed submitted version of manuscript: all authors. Approved the final version of the manuscript on behalf of all authors: Gokaslan. Statistical analysis: Darveau. Administrative/technical/material support: Darveau. Study supervision: Gokaslan, Darveau, Leary, Niu.

\section{Correspondence}

Ziya L. Gokaslan: Rhode Island Hospital, Providence, RI. ziya_gokaslan@brown.edu. 